\title{
An Objective Measure to Compare some Automatic Generation Methods of NN Architectures
}

\author{
G. Gutiérrez, J.M. Molina, I. M. Galván, A. Sanchis \\ Departamento de Informática. Universidad Carlos III de Madrid. \\ Avda. de la Universidad, 30, 28911 (Leganés), Madrid, Spain \\ molina@ia.uc3m.es, \{ggutierr, igalvan, masm@inf.uc3m.es\}
}

\begin{abstract}
Many methods to codify Artificial Neural Networks have been developed to avoid the defects of direct encoding schema, improving the search into the solution's space. A method to evaluate how the search space is covered and how move along search process applying genetic operators is needed in order to evaluate the different encoding strategles for a kind of Artificial Neural Networks, Feedforward Neural Networks. A first step of this method is considered with two encoding strategles, a direct encoding method and an indirect encoding scheme based on cellular antomata.
\end{abstract}

Keywords: Neural Networks, Genetic Algorithms, Automatic Design in NN, Indirect Encoding Methods

\section{INTRODUCTION}

In the last years, many works have been centered toward automatic resolution of the design of neural network architectures $[1,2,3,4,5,6]$. Two representation approaches exist to find the optimum net architecture using Genetic Algorithms (GA): one based on the complete representation of all the possible connections and other based on an indirect representation of the architecture. The first one is called Direct Encoding Method, and is based on the codification of the complete network into the chromosome of the GA, and starts with the Ash's work [7] and continues with other works, including [8]. The second one is the called Indirect Encoding Method that consists on codifying, not the complete network, but a compact representation of it [1]. In 1990, Kitano [9] presents a new method for designing neural networks by means of Genetic Algorithms, where neural networks are represented through graph grammars, codified in the chromosomes of the individuals. The grammatical approach is not the only proposed representation method. Merril et al. [10] introduced a fractal representation for encoding the architectures, arguing that this representation is more related with biological ideas than constructive algorithms.

Indirect Encoding Methods are applied in order to reduce the length of the genotype, the search space, and to make the problem more scalable. No exhaustive analysis of those features exists in the literature. The main problem is the definition of an objective measure to evaluate different codifications and search strategies. The measure should be able to evaluate the efficiency of the search strategy, analyzing the different neural network architectures generated with several methods (see Figure 1).

Typically, a good problem representation requires the representation of any possible solution, and a good search strategy requires that similar genotypes produce similar architectures. In this way, all the search space is covered and the fitness function is able to guide the search. Figure 1 shows that this evaluation (representation and search) should be accomplished over the generated neural network architectures. In [11], a preliminary study of the representation capacity is presented. In this work, a function $\left(f:\{0,1\}^{n} \times\{0,1\}^{m} \rightarrow \mathfrak{K}\right)$ over the binary matrix domain is defined to obtain a real value that represents the matrix, and for extension each neural network architecture. The results of this work show that the indirect encoding method based on grammatical approach is able to cover the search space. However, it is not enough clear whether the results do show this, because many of the points are overlapping and there are large areas of neural networks space that have not been generated. Then, one more rigorous method of showing how much of the search space is covered by the encoding scheme might be to use Hamming distances between chromosomes networks (binary matrix) generated from random samples of genotypes (typically in Evolutionary Computation). Then the average distance over such a sample of genotypes could be compared with the average distances calculated over randomly generated genotypes.

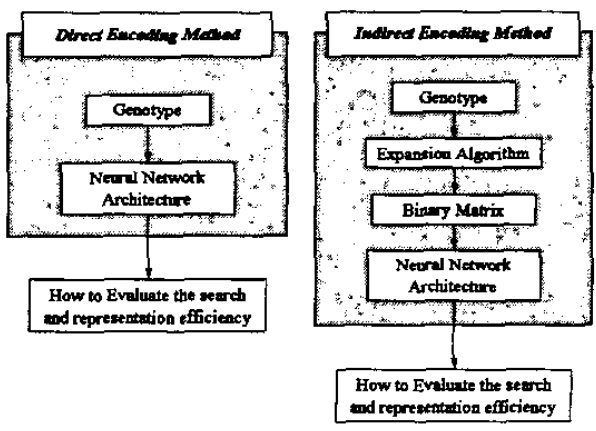

Figure 1. Description of the Comparison Problem between Direct and Indirect Encoding Methods.

The application of Hamming distances in each indirect encoding problem could not be apply because each chromosome and its corresponding binary matrix has a different meaning depending on the expansion method. We need an objective measure to evaluate in the same way the different methods. This objective measure could be used to evaluate the search space, because the major problem with indirect encoding schemes is often argued to be that the 
search space is highly rugged with comparison to direct encoding schemes, i.e. small changes to the genotype produce large changes in the network architecture and final network performance. This problem makes the schemes difficult to use in artificial evolutionary field.

In this work, a procedure (in three steps) defines an objective measure to evaluate the improvements of the indirect encoding methods. This measure is applied over two constructive encoding schemes based on direct and indirect encoding. In particular the selected indirect encoding method is based on Cellular Automata (CA) $[12,13]$. Using the procedure defined in this paper, we could analyze the generative capacity (representation) and the search space in the domain of genotypes of direct codification scheme. And compare with the indirect one.

In this paper some preliminary results about the first step of the procedure are shown. In these results the density function of the initial population of neural networks is analyzed.

Section 2 and 3 are related with the encoding schemes used in this work. Section 4 describes the objective function. The results of the probability distribution estimation are shown in section 5 . Finally some conclusions are presented.

\section{DIRECT ENCODING}

As it has been mentioned in the introduction, direct encoding methods are based on the codification of the complete network into the chromosome of the GA. Generally, the term direct encoding refers to encoding strategies that directly encode parameters of the neural networks such as weights, connections, etc, into the chromosome. Some systems focus on the weights encoding $[8,14,15]$, whereas others works focus on the architectures encoding $[13,16,17]$. There also systems that combine the two issues, so that both the architecture and weights are evolved at the same time [18, 19].

Our interest is focused in the evolution of the architectures; this is the number of hidden neurons and the connections between different layers, while the task of weight training is left to be carried out by learning rules, as the standard backpropagation algorithm.

The most usual way to codify an architecture using a direct encoding scheme is to specify into a gen of the chromosome the presence or absence of a connection in the network using 1 or 0 , respectively [2]. Thus, the chromosome is a binary string and is written in terms of the interconnected structure or connections matrix of the neural network. The chromosome is just the concatenation of rows (or columns) of the connections matrix. That connections matrix is usually specified as an NxN binary matrix $C=\left(c_{i j}\right)_{N x N}$ where $N$ is the total number of neurons in the network and $c_{i j}$ indicates presence or absence of the connection from neuron $i$ to neuron $j$. In this case all the neurons in the network are enumerated and each position in the matrix represents the connection between each pair of neurons in the network. Constraints on architectures can be incorporated into such representation scheme by setting constraints in the matrix; for instance a feed forward neural network will have zero entries in the lower triangle of the matrix.

Since our work focus to design optimal feedforward architectures, the direct encoding scheme used in this work has been designed to just represent feedforward neural networks (FNN) with one hidden layer, rejecting any other type of neural network, as recurrent neural networks. The purpose of the direct scheme used in this work is to avoid codifying no useful positions of the connections matrix -as positions representing recurrent connections- into the genome. Thus, the length of the chromosome is reduced, which increases the efficiency of the evolution, as it is well known.

The direct encoding scheme used in this work is based on a binary matrix of dimension $\operatorname{Dim}_{x} \times \operatorname{Dim}_{y}$, where $\operatorname{Dim}_{x}$ is equal to the number of input neurons plus the number of output neurons and $\operatorname{Dim}_{y}$ corresponds with the maximum number of hidden neurons to be considered (see figure 2). To relate that matrix with an architecture of a FNN with one hidden layer, the meaning for the grid position $(i, j)$ is defined as follows. Let's $n$ the number of input neurons; if $i \leq n$ then $(i, j)$ represents a connection between the $i$-th input neuron and the $j-t h$ hidden neuron; if $i>n,(i, j)$ represents a connection between the $j$-th hidden neuron and the $(i-n)$-th output neuron. That relation is shown in figure 2 . The chromosome is just the concatenation of the matrix rows and DimxxDimy gives the length of the chromosome.

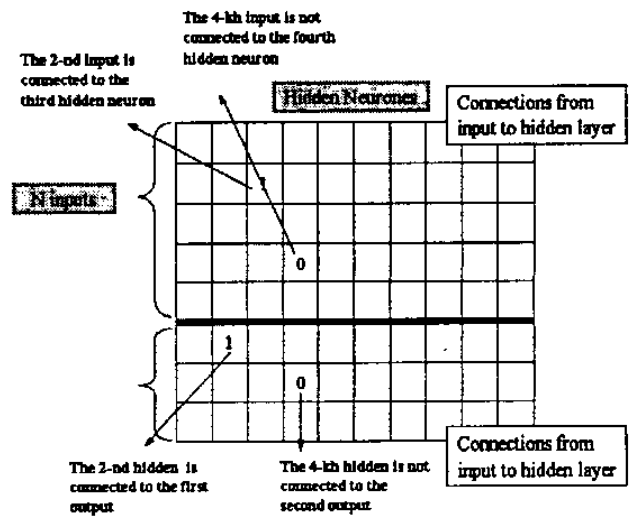

Figure 2. Relation between binary matrix and FFN architectures

For the direct encoding schemes presented in this section, standard genetic operations can be used to evolve the population of architectures. Other direct encoding schemes have been also appear in the literature, for instance [21], although in this case specific genetic operators must be defined. 


\section{INDIRECT ENCODING}

Direct encoding representations of neural network architectures are relatively simple, straightforward to implement and they would be suitable for problems requiring a small number of neurones and connections. They may allow rapid generation and optimisation of tightly pruned designs. However, the capabilities of direct encoding for solving large problems are limited, because large problems require much larger chromosome. In order to reduce the length of the genotype and to make the problem more scalable, indirect encoding schemes to represent neural network architectures have been proposed in the last years $[1,9,10]$. They basically consist of codifying, not the complete network, but a compact representation of it. That representation (for instance based on grammars, fractals, etc.) is expanded to obtain neural network architecture. The genetic algorithm evolves that representation, instead of connections matrix of neural networks as in the case of direct encoding (see figure 1).

The indirect encoding scheme used in this work is based on cellular automata. The system -shown in figure 3- is composed of three different modules: the Genetic Algorithm Module, the Cellular Module and the Neural Network module. The scheme is cycled, and each cycle corresponds to a genetic algorithm generation. By means of genetic operators a population of FNN architectures (individuals expressed through cellular automata) is obtained, except the first one, that is randomly generated as is usual. These individuals are evaluated through a fitness function. To calculate the fitness value, the cellular automata are executed over an initial configuration codified in the individual chromosome, and a binary matrix is obtained. In a next step, this matrix is translated into a FNN architecture and is trained. After the FNN is trained, is tested and an error value is obtained. With this error and some other relevant information about the FNN (size, learning cycles, etc.), the fitness value of the considered individual is computed. The process is repeated until all the population is evaluated. The proposed architecture follows the general mechanism of other well-known systems like Kitano's [9] and the GANET system $[11,20]$.

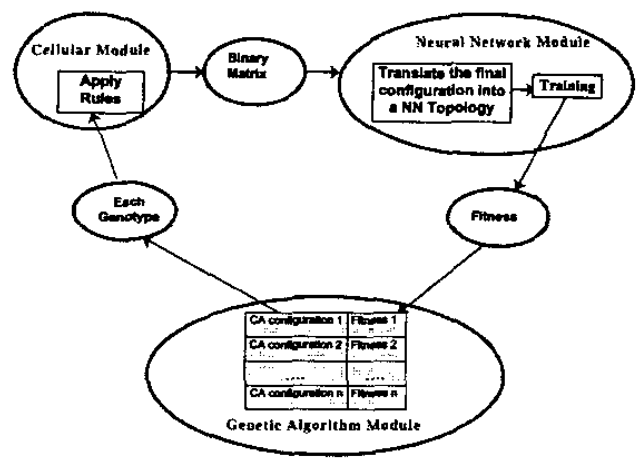

Figure 3. Cellular System's architecture and modules relationship.
The cellular module is based on the cellular automata philosophy and is composed of two bi-dimensional cellular systems. Several seeds give the initial configurations of the two-dimensional cellular systems and the rules of these systems are applied to generate final configurations. The first cellular system, called growing cellular automata, is related with the generation of FNN with a large number of connections. The second cellular system, named decreasing cellular automata, is related with the reduction of this number of connections. The seeds are defined through two coordinates and they are codified in the chromosome of the genetic algorithm, which evolves to maximize the performance of FNN architecture A detailed description of the system can be founded in [13]. The motivation of this approach is based on the idea that only a few seeds for initial configurations of cellular systems can produce a wide variety of FNN architectures. Thus, the length of the chromosome is reduced.

\section{AN OBJETIVE MEASURE TO COMPARE AUTOMATIC GENERATION METHODS}

The goal of the system is to evaluate different encoding algorithms. In this way, we need to evaluate the neural networks generated to analyze the generative capacity (how many different architectures the method is able to generate) over the whole search space and the search strategy (how the Genetic Algorithm generates neural networks).

In order to analyze the behavior of the different encoding methods, three kind of information have to be studied:

1. The first one is related with the ability of the methods to generate an initial population that covers the complete search space. As the initial population has a finite size, the individuals are a subset of the search space. Clearly, an algorithm that generates random individuals following an uniform distribution over the search space is better than algorithms that introduce a bias in the initial population.

2. The second one is related with the way the algorithm search in the search space. The best situation to apply genetic algorithm is that small changes in the genotype produce small changes in the fitness value. This situation avoids the epistasis problem. This problem could not be addressed directly (as in other genetic approximations) with the indirect encoding methods because of the particular process introduced to translate the genotype in to the neural network.

3. The third one is related with the special search space that represents the neural networks. In this space, different binary matrix could represent the same neural network. Then a mathematical function will be required to identify similar architectures and to represent clearly the above points.

We propose to approximate the points one and two, analyzing the number of hidden neurons $\left(\mathrm{n}_{\mathrm{h}}\right)$ and the number of connections $\left(n_{c}\right)$. We propose to calculate the histogram of $n_{h}$ and $n_{c}$ for the initial population (point one), and to evaluate random walks (representing in a 3-D graph the 
fitness landscape over $n_{b}$ and $n_{c}$ ) for the search (point two). For the last point, we propose a method based on a unique codification of the generated neural network architecture. Then, a function $\left(f:\{0,1\}^{n} \times\{0,1\}^{m} \rightarrow\{0,1\}^{n^{\prime}} \times\{0, I\}^{m}\right)$ over the binary matrix domain is defined to obtain a binary matrix that represents in the same way, any binary matrix (and for extension each neural network architecture) generated using any method. Really, this function codifies again the generated $\mathrm{NN}$, and, so, we can choose as function $f$ the direct codification of a NN (see Figure 4).

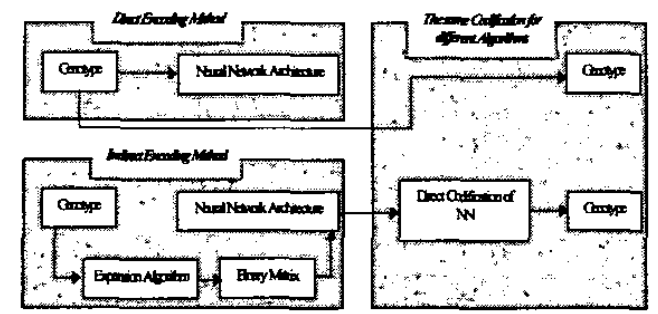

Figure 4. Description of the Objective Measure.

In this work, results concerning to the first point are shown, in order to study the ability of encoding algorithms to cover the architecture. The follows several steps we present the results of the analysis of the initial population. The study follows several steps:

1. Generate a random initial population of chromosomes with an uniform distribution.

2. Apply the translation process for each encoding method and for each chromosome to obtain the correspondent binary matrix or neural network architecture.

3. Make a histogram of the number of hidden neurons $\left(n_{h}\right)$ in the binary matrix.

4. Find the density function that corresponds with the histogram of $n_{\mathrm{b}}$.

5. Make a histogram of connections $\left(n_{c}\right)$ in the binary matrix.

6. Find the density function that corresponds with the histogram of $n_{c}$

7. Finally, analyze the 3-D histogram (considering the bidimensional space $n_{b} \times n_{c}$ ) to find the corresponding density function.

The probability density function allows estimating the generation probability of a neural network with a specific number of hidden neurons and connections. This information shows how each encoding method covers the search space in the initial population.

\section{ANALYSIS OF INITIAL POPULATION DISTRIBUTION}

For both encoding methods an initial population of $10^{5}$ chromosomes are randomly generated. In both encoding schemes every value for an element of the chromosome has the same probability than the other values. With direct encoding method, in any element of the binary matrix (the chromosome) there is a " 0 " or a " 1 " with the same probability $(p=1 / 2)$. For indirect coding scheme based on $\mathrm{CA}$ the elements of the chromosome are integers ranging in value from 0 to $\left(n_{t}+n_{o}-1\right)$, where $n_{I}$ and $n_{o}$ are the number of inputs and outputs respectively that order, fixed both by the problem that FNN resolves. As in direct encoding for an element of the chromosome, all the values have the same probability, $1 /\left(n_{i}+n_{o}\right)$.

In these preliminary results a generic domain is used, with two inputs and two outputs, $\left(n_{I}=n_{O}=2\right)$. Then the maximum number of hidden neurons of FNN considered is $\left(n_{I}+n_{o}\right)^{2}=16$, [13]. Therefore the length of the chromosome for direct encoding scheme is 64 , equal to the maximum number of connection considered. The length of the chromosome for indirect encoding scheme based on CA depends only on the number of growing seeds ( $g s$, related to growing cellular automata) and decreasing seeds ( $g d$, related to decreasing cellular automata) used. In this paper the results for $4 \mathrm{gs}$ and $4 d s$ will be exposed in histograms. Results for other number of seeds are similar.

For both encoding scheme when the binary matrix is obtained, if a column with all they values are " 0 " it means that the hidden node that this column represents has not any connection from input layer or to output layer. And it could be not considered, i.e. it could be eliminated from the FNN.

When all chromosomes are translated into his phenotype, i.e. a FNN, how many have the same number of hidden nodes $\left(\mathrm{n}_{\mathrm{h}}\right)$, Figure 5 and Figure 6, and how many have the same number of connections (nc), Figure 7 and Figure 8 , for direct and indirect scheme are shown.

Finally in Figure 9 and Figure 10 the 3-D histograms for both encoding schema is shown. In them, the number of FNN with a same number of hidden nodes and a same number of connections is displayed.

About the histogram shown in Figure 5 and Figure 6, for number of FNN with the same number of hidden neurons, it is clear that for direct encoding scheme the probability density function (p.d.f.), corresponding to this discrete distribution, could be obtained exactly from the probability that a column has no element equal to "1". And the same for histogram of FNN with the same number of connections in the binary matrix. It is clear that must be a binomial distribution. But for indirect encoding it not feasible, at least too complex, to obtain exactly the discrete probability density function that corresponding to the histogram of $n_{c}$ and $n_{h}$.

As it is observed in figures, it is clear that indirect encoding scheme cover the search space for a initial random population so much better than the direct encoding method if a random, initial population is generated. The probability to obtain a FNN from the initial population randomly generated with direct encoding scheme with less hidden neurones than 10 is very low. The same for FNN full connected or with a high 
percentage of connections, taking into consideration that the maximum number of connections for a given number of hidden nodes $(h)$ is $\left(h \cdot\left(n_{I}+n_{o}\right)\right)$. However for the initial population obtained with indirect encoding scheme based on CA, FNN with hidden nodes ranging in value from 2 to 16 (i.e. maximum of hidden nodes) are obtained. And on the contrary than direct encoding, FNN with percentages of connectivity ranging from minimum $\left(10 \% / n_{i}+n_{o}\right)$ to maximum are obtained, Figure 11 and Figure 12. All these advantages for indirect encoding scheme could make the search more feasible for the evolutionary algorithm.

\section{CONCLUSIONS AND FUTURE WORKS}

Indirect Encoding methods are applied in order to reduce the length of chromosomes. As a Evolutionary Algorithm begins the search with a random initial population, it is important that it covers as completely as possible the search space. The analysis presented in this work shows that direct encoding methods do not carry out this condition, on the contrary than the indirect encoding scheme based on CA aforesaid.

In future works, estimations of what density distribution functions correspond with the histograms shown will be study. Random walks over the space of chromosomes will be evaluated in order to get to know how the genetic algorithm look for in the search space for both encoding methods, direct and indirect.

\section{REFERENCES}

[1] S. Harp, Samad T. and Guha. A. Towards the Genetic Synthesis of Neural Networks. Proceedings of the Third International Conference on Genetic Algorithms and their applications, pp 360-369, San Mateo, CA, USA, 1989.

[2] G.F. Mitler, P.M. Todd and S.U. Hegde. Designing neural networks using genetic algorithms. In Proc. of the third international conference on genetic algorithrns and their applications, pp 379-384, San Mateo, CA, USA, 1989.

[3] S. Harp, Samad T. and Guha A. Designing ApplicationSpecific Neural Networks using the Genetic Algorithm, Advances in Neural Information Processing Systems, vol2, 447-454, 1990.

[4] F. Gruau. Genetic Synthesis of Boolean Neural Networks with a Cell Rewriting Developmental Process. Proc. of COGANN-92 International Workshop on Combinations of Genetic Algorithms and Neural Networks, pp. 55-74, IEEE Computer Society Press, 1990

[5] F. Gruau. "Neural Network Synthesis Using Cellular Encoding and the Genetic Algorithm". Ph.D. Thesis, Ecole Normale Supèrieure de Lyon, (1994).

[6] F. Gruau. Automatic Definition of Modular Neural Networks. Adaptive Behavior, vol. 2, 3, 151-183, 1995.

[7] T. Ash. Dynamic Node Creation in Backpropagation Networks ICS Report 8901, The Institute for Cognitive Science, University of California, San Diego (Saiensu-sh, 1988), 1988.

[8] D.B. Fogel, Fogel L.J. and Porto V.W. Evolving Neural Network, Biological Cybernetics, 63, 487-493, 1990.

[9] H. Kitano. Designing Neural Networks using Genetic Algorithms with Graph Generation System, Complex Systems, 4, 461-476, 1990.

[10] J.W.L. Merril and R.F. Port. Fractally configured Neural Networks. Neural Networks, 4, 53-60, 1991.
[11] M. A. Guinea, G. Gutierrez, I. Galván, A. Sanchis, J. M Molina Generative Capacities of Grammars Codification for Evolution of NN Architectures. International Conference on Evolutionary Computation, WCCI, 2002. USA.

[12] J.M. Molina, I. Galván, P. Isasi, A. Sanchis. Grammars and Cellular Automata for Evolving Neural Networks Architectures. IEEE International Conference on Systems, Man and Cybernetics, 2001. USA.

[13] G. Gutierrez, I. M.Galván, A. Sanchis, J. M. Molina. Generative Capacities of Cellular Auomata Codification fo Evolution of NN Codification. To appear in International Conference on Artificial Neural Networks 2002

[14] Whitley, D., Starkweather, T., Bogart C., 1990, Genetic Algorithms and Neural Networks: Optimizing Connections and Connectivity, Parallel Computing, 14, pp. 347-361.

[15] Montana, D. J. and Davis, L. D.: Training feedforward networks using genetic algorithms. In Proceedings of the International Joint Conference on Artificial Intelligence, San Mateo, CA, 1989. Morgan KaufnRNA.

[16] J.D. Schaffer, R.A. Caruana and L.J. Eshelman. Using genetic search to exploit the emergent behaviour of neural networks. Physica D, 42, pp 244-248, 1990

[17] Alba, E., Aldana, J. F., and Troya, J. M.: Fully automatic RNA design: a genetic approach. In Proc. of In't Workshop on Artificial Neural Networks (IWRNA'93), 399-404. SpringerVerlag, 1993

[18] J.R. Koza and J.P. Roce. Genetic generation of both weights and architecture fro a nueral network. In Proc. Of IEEE Int. Joint Conf. On Neural Networks, 2, 667-673, 1991

[19] D.W. White. GANNet: A genetic algorithm for searching topology and weight spaces in neural networks design. PhD Thesis, University of Maryland, 1993.

[20] Molina, J. M., Torresano, A., Galván, I. M., Isasi, P., Sanchis, A.: Evolution of Context-free Grammars for Designing Optimal Neural Networks Architectures. GECCO 2000. Workshop on Evolutionary Computation in the Development of ANN. USA. Julio, 2000

[21] W. Schiffmann, M. Joost, and R. Werner. Synthesis and performance analysis of multilayer neural network architectures. Technical Report 16/1992, University of Koblenz, Institute fur Physics, Rheinau 3-4, D-5400 Koblenz, 1992. http://citeseer.nj.nec.com/schiffmann92synthesis.html

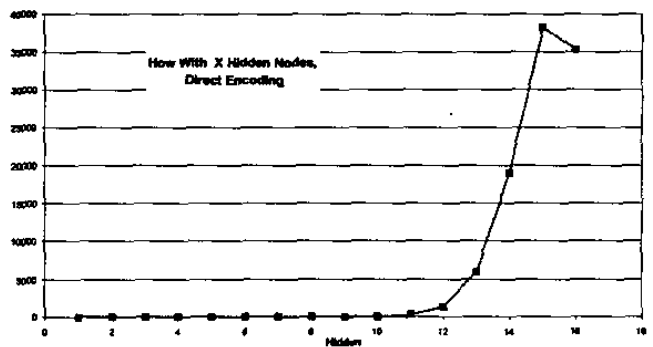

Figure 5. How many FNN have the same number of hidden nodes, Direct Encoding. 


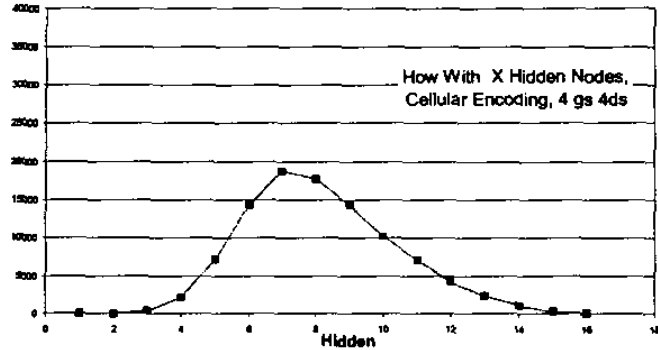

Figure 6 . How many have the same number of hidden nodes, Indirect Encoding.

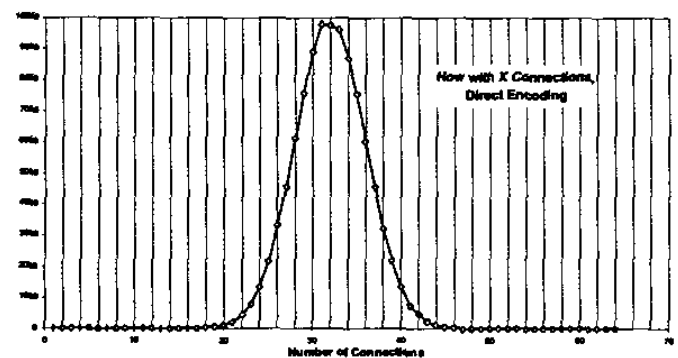

Figure 7. How many have the same number of connections, Direct Encoding.

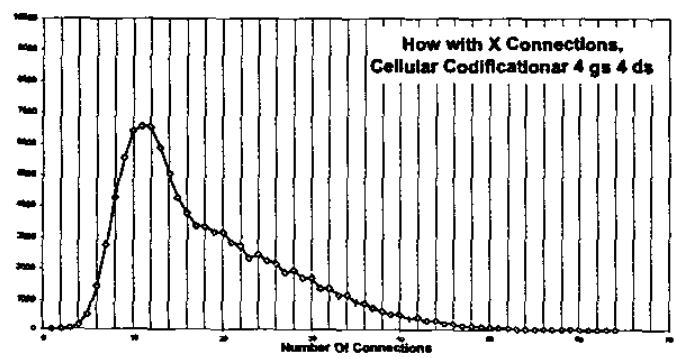

Figure 8. How many have the same number of connections, Indirect Encoding.

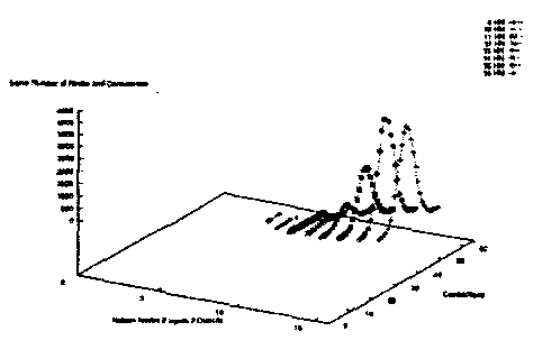

Figure 9. How many have the same number of hidden nodes and connections, Direct Encoding.

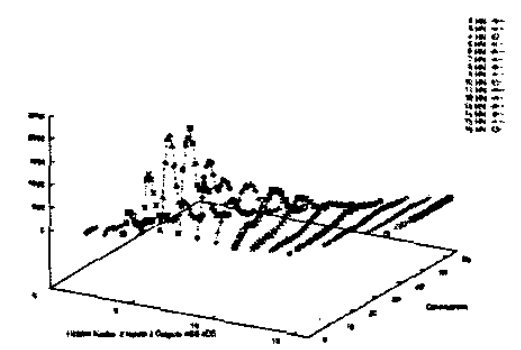

Figure 10. How many have the same number of hidden nodes and connections, Indirect Encoding.

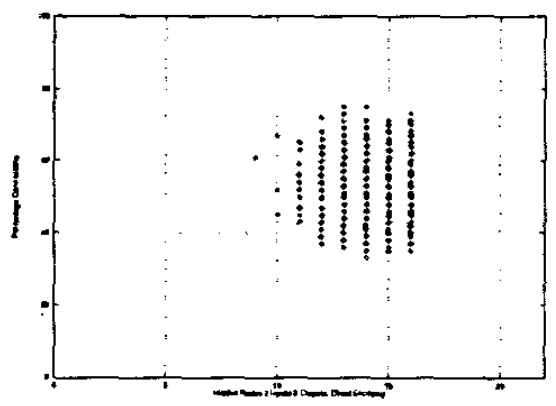

Figure 11. Percentage of connectivity, Direct Encoding

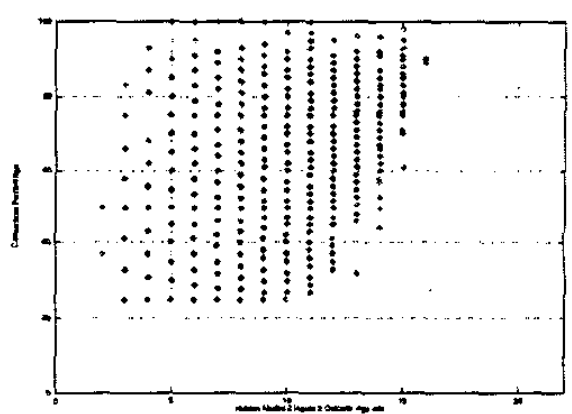

Figure 12. Percentage of connectivity, Indirect Encoding 